\title{
Probabilidade de ocorrência de déficit hídrico na região de Dourados, MS
}

\footnotetext{
${ }^{1}$ Embrapa Agropecuária Oeste, CP 661, CEP 79804-970, Dourados, MS. Fone: (67) 425-5122. Email: fietz@cpao.embrapa.br (Foto)

2 Embrapa Agropecuária Oeste, Fone: (67) 425-5122. Email: urchei@cpao.embrapa.br

${ }^{3}$ Departamento de Engenharia Rural, ESALQ-USP, CP 09, CEP 13418-900, Piracicaba, SP. Email: frizonne@carpa.ciagri.usp.br
}

Protocolo $028-8 / 3 / 2001$

\begin{abstract}
Resumo: Este trabalho foi elaborado com o propósito de se determinar a probabilidade de ocorrência de déficit hídrico na região de Dourados, MS. O estudo baseou-se em dados diários de evapotranspiração e de precipitação pluviométrica de um período de aproximadamente 20 anos. A precipitação e os elementos meteorológicos utilizados na estimativa da evapotranspiração, foram coletados na Estação Agrometeorológica da Embrapa Agropecuária Oeste, em Dourados. A evapotranspiração de referência foi estimada pelo método FAO Penman-Montheith. $O$ déficit hídrico diário foi determinado através de um balanço hídrico seqüencial, enquanto os valores totais de déficit hídrico de cada decêndio formaram séries que foram ajustadas à distribuição normal. Através da função de freqüência acumulada, geraram-se valores de déficit hídrico para períodos de retorno de 3, 4, 5, 10 e 15 anos. Com base na análise dos dados, verificou-se que as menores e maiores probabilidades de déficit hídrico ocorrem de abril a julho e de agosto a setembro, respectivamente, enquanto de outubro a janeiro também podem ocorrer altos índices de déficit hídrico, justificando, tecnicamente, o uso da irrigação em caráter complementar, na região de Dourados.
\end{abstract}

Palavras-chave: balanço hídrico, evapotranspiração, precipitação

\section{Probability of the ocurrence of water deficits in Dourados, MS - Brazil}

\begin{abstract}
The aim of this work was to determine the probability of the occurrence of water deficits in Dourados, in Mato Grosso do Sul State, Brazil. The study was based on daily data of evapotranspiration and rainfall for a period of about 20 years. The reference evapotranspiration was estimated by the FAO Penman-Montheith method. The daily water deficit was determined through a sequential water balance. The total water deficit values of 10 day periods resulted in series that were adjusted to normal distribution. By means of the accumulated frequency function, values of water deficit were generated for return periods of $3,4,5,10$ and 15 years. The lowest and highest water deficit probabilities occur during April to July and August to September, respectively. Also, there is high water deficit probability occurrence during October to January, justifying technically the use of complementary irrigation in Dourados.
\end{abstract}

Key words: water balance, evapotranspiration, precipitation

\section{INTRODUÇÃO}

A região de Dourados, MS, situa-se numa das principais áreas de produção agrícola do Brasil, onde é cultivado, anualmente, mais de um milhão de hectares, principalmente com soja e milho. A ocorrência de déficit hídrico é uma das principais causas de perdas agrícolas na região, pois apenas uma pequena parte das lavouras é dotada de irrigação. Uma forma de se reduzir os prejuízos causados pelo déficit hídrico em culturas não irrigadas, é definir adequadamente as melhores épocas de plantio, possibilitando que os estádios mais críticos das plantas coincidam com os períodos menos propensos às estiagens ou veranicos. Como as previsões climáticas não alcançaram, ainda, a precisão desejada, principalmente a longo prazo, estimativas probabilísticas da ocorrência de déficit hídrico podem ser de grande utilidade.

O balanço hídrico é uma metodologia muito utilizada para se avaliar o armazenamento de água no solo e quantificar déficits e excessos hídricos ao longo do tempo. De acordo com Pereira et al. (1997) o balanço hídrico é um sistema contábil de monitoramento da água do solo e resulta da aplicação do princípio de conservação da massa em um volume de solo 
vegetado. A variação do armazenamento representa o balanço entre as entradas e saídas de água do volume de controle, em um intervalo de tempo.

Os resultados de um balanço hídrico podem ser utilizados no zoneamento agroclimático, na determinação da demanda hídrica potencial das culturas irrigadas, no planejamento da pesquisa e para identificar o regime hídrico de uma região (Aguilar et al., 1986). Em agricultura irrigada, o balanço hídrico é aplicado na determinação de períodos com excesso ou escassez de água e, principalmente, na quantificação das deficiências hídricas de uma região (Amorim Neto, 1989).

Reichardt (1987) faz uma distinção entre balanço hídrico real e climatológico. De acordo com o autor, o primeiro contabiliza as adições e retiradas de água em uma área de produção agrícola, fornecendo subsídios para definir quando e quanto irrigar. $\mathrm{O}$ balanço hídrico climatológico estima, com base em dados climáticos de vários anos, o que vai ocorrer e possibilita a caracterização hídrica de uma região.

O balanço hídrico climatológico, descrito por Thornthwaite \& Mather (1955), possibilita monitorar o armazenamento de água no solo e quantificar o excesso e a deficiência hídrica, sendo também uma ferramenta para se estudar a viabilidade de implantação de sistemas de irrigação e/ou drenagem, numa região (Pereira et al., 1997).

Em Londrina, PR, com base em um balanço hídrico diário seqüencial, Caramori \& Faria (1987) verificaram que os meses de agosto, setembro e abril, respectivamente, apresentam a maior freqüência de déficit hídrico, enquanto junho, dezembro, janeiro e fevereiro, são os que possuem melhor condição de umidade no solo. Utilizando a mesma metodologia, Caramori et al. (1991) observaram que em Cascavel, PR, os períodos mais secos ocorrem no final de agosto, início de setembro, janeiro fevereiro e abril, enquanto os meses de maio, junho, julho, outubro e novembro apresentam a maior disponibilidade hídrica.

O objetivo deste trabalho foi determinar a probabilidade de ocorrência de déficit hídrico na região de Dourados, com base em um balanço hídrico climatológico diário seqüencial.

\section{MATERIAL E MÉTODOS}

O trabalho baseou-se em dados diários de evapotranspiração de referência e de precipitação pluviométrica de um período de aproximadamente 20 anos (junho de 1979 a dezembro de 1998). A precipitação e os elementos meteorológicos utilizados na estimativa da evapotranspiração (temperatura e umidade relativa do ar, número de horas de brilho solar e velocidade do vento) foram coletados na Estação Agrometeorológica da Embrapa Agropecuária Oeste, de Dourados, MS, cujas coordenadas geográficas são: $22^{\circ} 14^{\prime} \mathrm{S}, 54^{\circ} 49^{\prime}$ W e altitude média de $452 \mathrm{~m}$. O clima da região é o Cwa (mesotérmico úmido, com verão chuvoso), de acordo com a classificação de Köppen.

A evapotranspiração de referência foi estimada pelo método FAO Penman-Monteith, conforme metodologia apresentada em Allen et al. (1998):

$$
\mathrm{ET}_{0}=\frac{0,408 \Delta \mathrm{R}_{\mathrm{n}}+\gamma \frac{900}{(\mathrm{~T}+273)} \mathrm{U}_{2}\left(\mathrm{e}_{\mathrm{s}}-\mathrm{e}_{\mathrm{a}}\right)}{\Delta+\gamma\left(1+0,34 \mathrm{U}_{2}\right)}
$$

em que:

$$
\begin{array}{ll}
\mathrm{ET}_{0} & \text { - evapotranspiração de referência, } \mathrm{mm} \mathrm{d}^{-1} \\
\mathrm{R}_{\mathrm{n}} & \text { - saldo de radiação, } \mathrm{MJ} \mathrm{m} \mathrm{m}^{-2} \mathrm{~d}^{-1} \\
\mathrm{~T} & \text { - temperatura média do ar a } 2 \mathrm{~m},{ }^{\circ} \mathrm{C} \\
\mathrm{U}_{2} & \text { - velocidade média do vento a } 2 \mathrm{~m}, \mathrm{~m} \mathrm{~s}^{-1} \\
\mathrm{e}_{\mathrm{s}} & \text { - pressão de saturação do vapor d'água, } \mathrm{kPa} \\
\mathrm{e}_{\mathrm{a}} & \text { - pressão atual do vapor d'água, } \mathrm{kPa} \\
\Delta & \text { - tangente da curva de pressão de saturação do vapor } \\
& \text { d'água no ponto correspondente à temperatura do ar, } \\
& \mathrm{kPa}^{\circ} \mathrm{C}^{-1} \\
\gamma & \text { - constante psicrométrica, } 0,0639 \mathrm{kPa}^{\circ} \mathrm{C}^{-1}
\end{array}
$$

O saldo de radiação foi estimado por:

$$
\mathrm{R}_{\mathrm{n}}=\mathrm{R}_{\mathrm{ns}}-\mathrm{R}_{\mathrm{nl}}
$$

sendo:

$$
\mathrm{R}_{\mathrm{ns}}=(1-\alpha) \mathrm{R}_{\mathrm{s}}
$$

$\mathrm{R}_{\mathrm{nl}}=\sigma\left[\frac{\mathrm{T}_{\mathrm{Mk}}^{4}+\mathrm{T}_{\mathrm{mk}}^{4}}{2}\right]\left(0,34-0,14 \sqrt{\mathrm{e}_{\mathrm{a}}}\right)\left(1,35 \frac{\mathrm{R}_{\mathrm{s}}}{\mathrm{R}_{\mathrm{so}}}-0,35\right)$

em que:

$$
\begin{array}{ll}
\mathrm{R}_{\mathrm{ns}} & \text { - saldo de radiação de ondas curtas, } \mathrm{MJ} \mathrm{m} \mathrm{m}^{-2} \mathrm{~d}^{-1} \\
\mathrm{R}_{\mathrm{nl}} & \text { - saldo de radiação de ondas longas, } \mathrm{MJ} \mathrm{m}^{-2} \mathrm{~d}^{-1} \\
\mathrm{R}_{\mathrm{s}} & \text { - radiação solar global, } \mathrm{MJ} \mathrm{m} \mathrm{d}^{-2} \mathrm{~d}^{-1} \\
\alpha & \text { - albedo, } 0,23 \\
\sigma & \text { - constante de Stefan-Boltzmann, } 4,90310^{-9} \mathrm{MJ} \mathrm{m}^{-2} \mathrm{~d}^{-1} \\
\mathrm{~T}_{\mathrm{Mk}} & \text { - temperatura máxima do ar, } \mathrm{K} \\
\mathrm{T}_{\mathrm{mk}} & \text { - temperatura mínima do ar, } \mathrm{K} \\
\mathrm{R}_{\mathrm{so}} & \text { - radiação solar em dias sem nuvens, } \mathrm{MJ} \mathrm{m}^{-2} \mathrm{~d}^{-1}
\end{array}
$$

A radiação solar e a radiação solar em dias sem nuvens foram estimadas a partir da equação de Angströn (Allen et al., 1998):

$$
\begin{gathered}
\mathrm{R}_{\mathrm{s}}=\mathrm{R}_{\mathrm{a}}\left(\mathrm{a}+\mathrm{b} \frac{\mathrm{n}}{\mathrm{N}}\right) \\
\mathrm{R}_{\mathrm{so}}=(\mathrm{a}+\mathrm{b}) \mathrm{R}_{\mathrm{a}}
\end{gathered}
$$

em que:

$\mathrm{Ra}$ - radiação solar no topo da atmosfera, $\mathrm{MJ} \mathrm{m} \mathrm{m}^{-2} \mathrm{~d}^{-1}$

n - número de horas de brilho solar diário

$\mathrm{N}$ - número de horas de brilho solar máximo

Os coeficientes da equação de Angströn, considerados constantes ao longo do ano, foram obtidos da recomendação de ABEAS (1998) para locais com latitudes de $0 \mathrm{a} 60^{\circ}: \mathrm{b}=0,52$ e a $=0,29 \operatorname{Cos} \phi=0,27$, em que $\phi$ é a latitude.

A radiação no topo da atmosfera foi estimada por:

$\mathrm{R}_{\mathrm{a}}=\frac{24(60)}{\pi} \mathrm{G}_{\mathrm{sc}} \mathrm{d}_{\mathrm{r}}\left[\omega_{\mathrm{s}} \operatorname{Sen}(\varphi) \operatorname{Sen}(\delta)+\operatorname{Cos}(\varphi) \operatorname{Cos}(\delta) \operatorname{Sen}\left(\omega_{\mathrm{s}}\right)\right]$ 
sendo:

$$
\begin{aligned}
& \mathrm{d}_{\mathrm{r}}=1+0,033 \operatorname{Cos}\left(\frac{2 \pi}{365} \mathrm{~J}\right) \\
& \delta=0,409 \operatorname{Sen}\left(\frac{2 \pi}{365} \mathrm{~J}-1,39\right) \\
& \omega_{\mathrm{s}}=\arccos [-\tan (\varphi) \tan (\delta)]
\end{aligned}
$$

em que:

$$
\begin{array}{ll}
\mathrm{G}_{\mathrm{sc}} & \text { - constante solar, } 0,0820 \mathrm{MJ} \mathrm{m}^{-2} \mathrm{~min}^{-1} \\
\mathrm{~d}_{\mathrm{r}} & \text { - distância relativa Terra-Sol } \\
\omega_{\mathrm{s}} & \text { - ângulo horário do pôr-do-sol, rad } \\
\varphi & \text { - latitude, rad } \\
\delta & \text { - declinação solar-pôr-do-sol, rad } \\
\mathrm{J} & \text { - dia juliano }
\end{array}
$$

A velocidade média do vento a $10 \mathrm{~m}\left(\mathrm{U}_{10}\right)$ foi convertida para $2 \mathrm{~m}$ de altura, através da seguinte relação (Allen et al., 1998):

$$
\mathrm{U}_{2}=\mathrm{U}_{10} \frac{4,87}{\ln (67,8(10)-5,42)}
$$

O déficit e o excesso de água foram determinados através de um balanço hídrico climatológico diário seqüencial, realizado segundo a metodologia de Thornthwaite \& Mather (1955). Considerou-se que, no início do balanço hídrico, o solo estava com armazenamento pleno.

A disponibilidade hídrica do solo na capacidade de campo, estimada em $56,0 \mathrm{~mm}$, foi calculada com base em dados de umidade nas tensões $10 \mathrm{kPa}\left(0,418 \mathrm{~cm}^{3} \mathrm{~cm}^{-3}\right)$ e $1,5 \mathrm{MPa}$ $\left(0,362 \mathrm{~cm}^{3} \mathrm{~cm}^{-3}\right)$ de 144 curvas de retenção de um Latossolo Roxo (Fietz, 1998) uma das principais unidades pedológicas da região de Dourados. A profundidade do sistema radicular foi considerada de 1,0 m, valor recomendado por Mota (1987) para pastagens.

\section{RESULTADOS E DISCUSSÃO}

De maneira geral, as diferenças entre médias e medianas foram pequenas, indicando que os valores de déficit hídrico apresentaram distribuições relativamente simétricas (Tabela 1) comportamento este comprovado pelo ajuste de todas as séries à distribuição normal, com os valores de máxima divergência do teste Kolmogorov-Smirnov, inferiores aos definidos para o nível de significância de 5\%.

Os coeficientes de variação dos dados foram muito altos, superiores a $50 \%$, evidenciando a grande variabilidade dos

\begin{tabular}{|c|c|c|c|c|c|c|c|}
\hline Decên. ${ }^{1}$ & Mín. ${ }^{2}$ & Máx. ${ }^{3}$ & $\begin{array}{l}\text { Méd. }^{4} \\
(\mathrm{~mm})\end{array}$ & Med..$^{5}$ & $\mathrm{DP}^{6}$ & $\begin{array}{l}\mathrm{CV}^{7} \\
(\%)\end{array}$ & $\mathrm{D}^{8}$ \\
\hline \multicolumn{8}{|c|}{ Janeiro } \\
\hline $01-10$ & 1,4 & 45,4 & 20,3 & 15,9 & 13,7 & 67,5 & 0,19 \\
\hline $11-20$ & 0,0 & 37,9 & 15,2 & 13,0 & 12,6 & 82,9 & 0,21 \\
\hline $21-31$ & 0,9 & 60,6 & 16,2 & 9,7 & 15,4 & 95,1 & 0,22 \\
\hline \multicolumn{8}{|c|}{ Fevereiro } \\
\hline $01-10$ & 3,1 & 34,7 & 11,7 & 10,3 & 8,9 & 76,1 & 0,19 \\
\hline $11-20$ & 1,0 & 25,5 & 12,5 & 13,5 & 6,8 & 54,4 & 0,11 \\
\hline $21-28$ & 0,7 & 30,8 & 9,6 & 5 & 9,7 & 101,0 & 0,21 \\
\hline \multicolumn{8}{|c|}{ Março } \\
\hline $01-10$ & 1,4 & 36,2 & 14,7 & 14,9 & 10,2 & 69,4 & 0,13 \\
\hline $11-20$ & 2,4 & 26,4 & 14,5 & 13 & 8,5 & 58,6 & 0,16 \\
\hline $21-31$ & 2,2 & 40,4 & 15,1 & 14,4 & 10,6 & 70,2 & 0,15 \\
\hline \multicolumn{8}{|c|}{ Abril } \\
\hline $01-10$ & 1,8 & 47,0 & 14,1 & 13,5 & 10,9 & 77,3 & 0,26 \\
\hline $11-2$ & 2,4 & 3 & 11,0 & & 7,9 & 1,8 & 0,14 \\
\hline $21-30$ & 0,9 & 38,3 & 10,9 & 9,2 & 9,3 & 85,3 & 0,18 \\
\hline \multicolumn{8}{|c|}{ Maio } \\
\hline $01-10$ & 0,9 & 28,1 & 12,4 & 10,5 & 9,3 & 75,0 & 0,16 \\
\hline $11-20$ & 0,3 & 24,3 & 7,7 & 4, & ,3 & 94,8 & 0,22 \\
\hline \multicolumn{8}{|c|}{ Junho } \\
\hline $01-10$ & 0,4 & 20,6 & 5,9 & 4,9 & 4,6 & 78,0 & 0,25 \\
\hline $11-20$ & & 17,8 & 8,2 & 7,8 & 5 & 67,1 & 0,12 \\
\hline $21-30$ & 0,9 & 21,8 & 9,1 & 9,1 & 5,6 & 61,5 & 0,14 \\
\hline \multicolumn{8}{|c|}{ Julho } \\
\hline $01-10$ & 2.0 & 41,3 & 11,1 & 10,3 & 8,9 & 80,2 & 0,18 \\
\hline & & & & & & 9,2 & 0,12 \\
\hline $21-31$ & 0,9 & 34,3 & 19,0 & 20 & 11,0 & 57,9 & 0,17 \\
\hline \multicolumn{8}{|c|}{ Agosto } \\
\hline $01-10$ & 2,4 & 37,0 & 17,5 & 18,9 & & 54,3 & 0,13 \\
\hline $11-20$ & 1,0 & 45,7 & 20,1 & 19 & 12 & 59,7 & 0,11 \\
\hline $21-31$ & 1,6 & 57,4 & 27,3 & 27,4 & 16,2 & 59,3 & 0,16 \\
\hline \multicolumn{8}{|c|}{ Setembro } \\
\hline $01-10$ & 0,7 & 55,6 & 23,0 & 22,6 & 172 & 74,8 & 0,10 \\
\hline & & & 22,4 & & & 62,5 & 0,14 \\
\hline $21-30$ & 6,9 & 52,1 & 20,2 & 18,2 & 12,4 & 61,4 & 0,20 \\
\hline \multicolumn{8}{|c|}{ Outubro } \\
\hline $01-1$ & 3,8 & 43,3 & 18,7 & 17,7 & 12 , & 66,3 & 0,17 \\
\hline $11-20$ & 3,3 & 37,6 & 16,4 & 16,0 & 10,1 & 61,6 & 0,14 \\
\hline $21-31$ & 2,4 & 46,9 & 19,6 & 15,1 & 14,9 & 76,0 & 0,19 \\
\hline \multicolumn{8}{|c|}{ Novembro } \\
\hline $01-10$ & 2,0 & 42,3 & 15,9 & 14,3 & 10,6 & 66,7 & 0,16 \\
\hline & 2,8 & & 17,9 & 18,0 & & 62,6 & 0,16 \\
\hline $21-30$ & 2,3 & 40,0 & 17,4 & 12,7 & 12,4 & 71,3 & 0,19 \\
\hline \multicolumn{8}{|c|}{ Dezembro } \\
\hline $01-1$ & 1,0 & 54, & 18,2 & 16,0 & 14 & 78,0 & 0,15 \\
\hline & 0,4 & 43,0 & 14,0 & 11,9 & 11, & 78,6 & 0,17 \\
\hline $21-31$ & 4,0 & 64,0 & 21,4 & 13,1 & 18,8 & 87,8 & 0,2 \\
\hline
\end{tabular}
valores de déficit hídrico decendial do período.

Através da função de freqüência acumulada da distribuição normal, foram gerados valores de déficit hídrico decendial para períodos de retorno de três, quatro, cinco, dez e quinze anos ou, respectivamente, níveis de probabilidade de 67, 75, 80, 90 e $93 \%$ (Tabela 2). A interpretação das informações contidas nesta tabela pode ser feita da seguinte forma: considerando-se dez
Tabela 1. Estatística descritiva dos dados de déficit hídrico decendial, na região de Dourados, MS

${ }^{1}$ Decênio; ${ }^{2}$ Mínimo; ${ }^{3}$ Máximo; ${ }^{4}$ Média; $;{ }^{5}$ Mediana; ${ }^{6}$ Desvio padrão; $;{ }^{7}$ Coefíciente de variação $;{ }^{8}$ Valores de máxima divergência do teste Kolmogorov-Smirnov. $\mathrm{O}$ valor crítico para um nível de significância de $5 \%$ é igual a 0,29 (20 valores)

anos como período de retorno, existem $90 \%$ de probabilidade de que o déficit hídrico no primeiro decêndio de janeiro seja igual ou inferior a $37,9 \mathrm{~mm}$ ou, ainda, para o mesmo período, em apenas um de cada dez anos, o valor de déficit hídrico será igual ou superior a $37,9 \mathrm{~mm}$.

Na Figura 1 são apresentados os valores médios decendiais de precipitação e evapotranspiração de referência da região de Dourados, com base nesta figura e na Tabela 2, é possível analisar-se o comportamento do déficit hídrico no transcorrer do ano. 
Tabela 2. Déficit hídrico decendial máximo esperado na região de Dourados, MS, para diferentes níveis de probabilidade e períodos de retorno

\begin{tabular}{|c|c|c|c|c|c|}
\hline \multirow{2}{*}{ Decêndio } & \multicolumn{5}{|c|}{ Nível de Probabilidade ${ }^{*}(\%)$} \\
\hline & 67 & 75 & 80 & 90 & 93 \\
\hline \multicolumn{6}{|c|}{ Janeiro } \\
\hline $01-10$ & 26,3 & 29,5 & 31,8 & 37,9 & 40,5 \\
\hline $11-20$ & 20,7 & 23,7 & 25,8 & 31,3 & 33,8 \\
\hline $21-31$ & 23,0 & 26,6 & 29,2 & 35,9 & 38,9 \\
\hline \multicolumn{6}{|c|}{ Fevereiro } \\
\hline $01-10$ & 15,6 & 17,7 & 19,2 & 23,1 & 24,8 \\
\hline $11-20$ & 15,5 & 17,1 & 18,2 & 21,2 & 22,5 \\
\hline $21-28$ & 13,9 & 16,1 & 17,8 & 22,0 & 23,9 \\
\hline \multicolumn{6}{|c|}{ Março } \\
\hline $01-10$ & 19,2 & 21,6 & 23,3 & 27,8 & 29,7 \\
\hline $11-20$ & 18,2 & 20,2 & 21,6 & 25,4 & 27,0 \\
\hline $21-31$ & 19,8 & 22,2 & 24,0 & 28,7 & 30,7 \\
\hline \multicolumn{6}{|c|}{ Abril } \\
\hline $01-10$ & 18,9 & 21,4 & 23,3 & 28,1 & 30,2 \\
\hline $11-20$ & 14,5 & 16,3 & 17,6 & 21,1 & 22,7 \\
\hline $21-30$ & 15,0 & 17,2 & 18,7 & 22,8 & 24,6 \\
\hline \multicolumn{6}{|c|}{ Maio } \\
\hline $01-10$ & 16,5 & 18,7 & 20,2 & 24,3 & 26,1 \\
\hline $11-20$ & 10,9 & 12,6 & 13,8 & 17,0 & 18,5 \\
\hline $21-31$ & 11,1 & 13,1 & 14,5 & 18,1 & 19,7 \\
\hline \multicolumn{6}{|c|}{ Junho } \\
\hline $01-10$ & 7,8 & 8,9 & 9,6 & 11,5 & 12,4 \\
\hline $11-20$ & 10,6 & 11,9 & 12,8 & 15,2 & 16,3 \\
\hline $21-30$ & 11,6 & 12,9 & 13,8 & 16,3 & 17,4 \\
\hline \multicolumn{6}{|c|}{ Julho } \\
\hline $01-10$ & 15,0 & 17,1 & 18,6 & 22,5 & 24,2 \\
\hline $11-20$ & 18,5 & 20,6 & 22,0 & 25,8 & 27,5 \\
\hline $21-31$ & 23,8 & 26,4 & 28,3 & 33,1 & 35,2 \\
\hline \multicolumn{6}{|c|}{ Agosto } \\
\hline $01-10$ & 21,7 & 23,9 & 25,5 & 29,7 & 31,5 \\
\hline $11-20$ & 25,4 & 28,2 & 30,2 & 35,5 & 37,8 \\
\hline $21-31$ & 34,4 & 38,2 & 40,9 & 48,1 & 51,2 \\
\hline \multicolumn{6}{|c|}{ Setembro } \\
\hline $01-10$ & 30,6 & 34,6 & 37,5 & 40,0 & 48,4 \\
\hline $11-20$ & 28,6 & 31,8 & 34,2 & 40,3 & 43,1 \\
\hline $21-30$ & 25,6 & 28,6 & 30,6 & 36,1 & 38,5 \\
\hline \multicolumn{6}{|c|}{ Outubro } \\
\hline $01-10$ & 24,1 & 27,1 & 29,1 & 34,6 & 37,0 \\
\hline $11-20$ & 20,8 & 23,2 & 24,9 & 29,3 & 31,3 \\
\hline $21-31$ & 26,1 & 29,6 & 32,1 & 38,7 & 41,6 \\
\hline \multicolumn{6}{|c|}{ Novembro } \\
\hline $01-10$ & 20,6 & 23,0 & 24,8 & 29,5 & 31,5 \\
\hline $11-20$ & 22,8 & 25,4 & 27,3 & 32,2 & 34,4 \\
\hline $21-30$ & 22,8 & 25,8 & 27,8 & 33,3 & 35,7 \\
\hline \multicolumn{6}{|c|}{ Dezembro } \\
\hline $01-10$ & 24,4 & 27,8 & 30,1 & 36,4 & 39,2 \\
\hline $11-20$ & 18,8 & 21,4 & 23,3 & 28,1 & 30,2 \\
\hline $21-31$ & 29,7 & 34,1 & 37,2 & 45,5 & 49,1 \\
\hline
\end{tabular}

* Os níveis de probabilidade 67, 75, 80, 90 e 93 correspondem, respectivamente, a períodos de retorno de $3,4,5,10$ e 15 anos

Considerando-se o mesmo nível de probabilidade ou período de retorno, pode-se observar que em abril, maio, junho e julho, principalmente do segundo decêndio de maio ao terceiro de junho, ocorrem os menores índices de déficit hídrico, apesar desses meses apresentarem baixos índices de precipitação (Figura 1), comportamento que pode ser atribuído à pequena demanda evapotranspirativa do período, resultante de temperaturas mais baixas e de menor número de horas de brilho solar e, conseqüentemente, de radiação líquida, que possibilita manter um relativo equilíbrio hídrico.

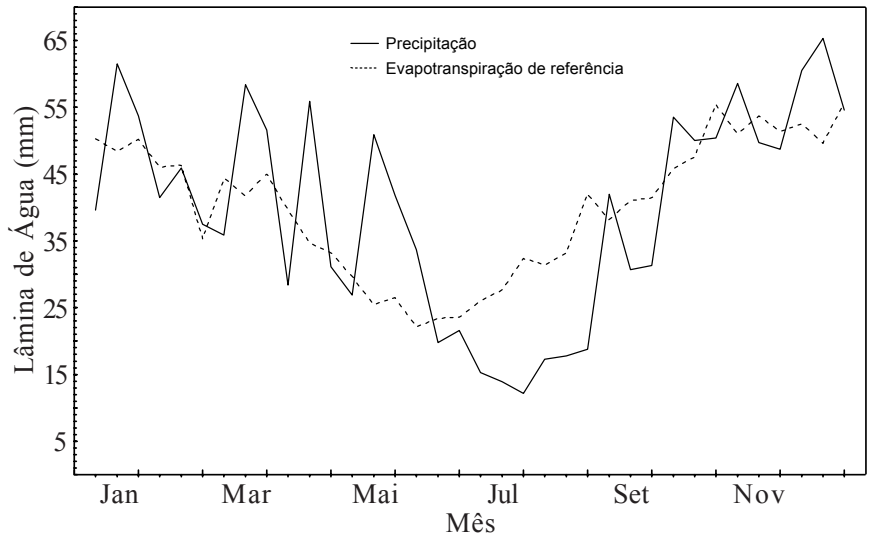

Figura 1. Valores médios decendiais de precipitação e de evapotranspiração de referência na região de Dourados, MS. Período de junho de 1979 a dezembro de 1998

No período que abrange o segundo decêndio de agosto até o terceiro de setembro, há maior probabilidade de ocorrerem os níveis mais elevados de déficit hídrico no ano. Em Dourados, assim como na região de Cascavel, PR (Caramori et al., 1991) os maiores índices de deficiência hídrica ocorrem principalmente no terceiro decêndio de agosto e no primeiro de setembro; portanto, deve-se evitar que os estádios mais sensíveis à escassez de água das culturas não irrigadas ocorram neste período. Apesar desses decêndios não apresentarem as maiores taxas de evapotranspiração, que ocorrem em novembro, dezembro e janeiro, esses resultados podem ser atribuídos aos baixos índices de precipitação do período (Figura 1).

De outubro a janeiro, também podem ocorrer valores altos de déficit hídrico, devido à grande demanda evapotranspirativa e à distribuição irregular das chuvas desses meses, nos quais, freqüentemente, são registrados veranicos e estiagens. Geralmente, nesses meses ocorrem os estádios fenológicos mais críticos das culturas de verão, justificando tecnicamente o uso da irrigação, em caráter complementar, na região de Dourados.

Os resultados diferem dos obtidos por Aguilar et al. (1986) que identificaram a existência de dois períodos bem definidos de deficiência hídrica na região de Dourados: janeiro a abril e junho a agosto, porém se deve ressaltar que esses autores realizaram o balanço hídrico para períodos mensais e decendiais e não consideraram a disponibilidade hídrica do solo.

\section{CONCLUSÕES}

1. Nos meses de abril a julho, principalmente do segundo decêndio de maio ao terceiro de junho, é prevista a ocorrência dos menores índices de déficit hídrico na região de Dourados.

2. O período que abrange o segundo decêndio de agosto até o terceiro de setembro, é o que apresenta a maior probabilidade de ocorrência dos níveis mais elevados de déficit hídrico.

3. De outubro a janeiro também pode haver valores altos de deficiência hídrica, justificando tecnicamente o uso da irrigação, em caráter complementar, na região de Dourados.

\section{LITERATURA CITADA}

ABEAS - Associação Brasileira de Educação Agrícola Superior. Curso de engenharia e manejo da irrigação. Módulo 2: Evapotranspiração: necessidade de água para as plantas cultivadas. Brasília: ABEAS; Viçosa: UFV, 1998. 181p. 
Aguilar, D.J.; Kruker, J.M.; Calheiros, R. de O.; Silva, C.A.S. da. Determinação da evapotranspiração potencial e balanço hídrico climático da região da Grande Dourados, MS. Dourados: EMBRAPA-UEPAE Dourados, 1986. 150p. Embrapa-UEPAE Dourados. Documentos, 21

Allen, R.G.; Pereira, L.S.; Raes, D.; Smith, M. Crop evapotranspiration: Guidelines for computing crop water requirements. Rome: FAO, 1998. 297p. FAO. Irrigation and Drainage Paper, 56

Amorim Neto, M. da S. Balanço hídrico segundo Thornthwaite \& Mather (1955). Petrolina: EMBRAPA-CPATSA, 1989. 18p. EMBRAPA-CPATSA. Comunicado Técnico, 34

Caramori, P. H; Faria, R. T. de. Freqüência de períodos de 10 dias consecutivos com deficiência hídrica (veranicos) para Londrina e Ponta Grossa. Londrina: IAPAR, 1987. 24p. IAPAR. Boletim Técnico, 20
Caramori, P. H; Oliveira, D. de; Faria, R. T. de. Freqüência de ocorrência de períodos com deficiência hídrica (veranicos) no Estado do Paraná. Londrina: IAPAR, 1991. 40p. IAPAR. Boletim Técnico, 36

Fietz, C.R. Variabilidade espacial do armazenamento de água no solo, visando ao manejo da irrigação por aspersão. Piracicaba: ESALQ, 1998.97p. Tese Doutorado

Mota, F. S. da. Balanço hídrico. In: Mota, F. S. da. Meteorologia agrícola. São Paulo: Nobel, 1987. p.279-309.

Pereira, A.R.; Vila Nova, N.A.; Sediyama, G.C. Evapo(transpi)ração. Piracicaba: FEALQ, 1997. 183p.

Reichardt, K. A água em sistemas agrícolas. São Paulo: Editora Manole, 1987. 188p.

Thornthwaite, C.W.; Mather, J.R. The water balance. Centerton: Drexel Institute of Technology, 1955. 104p. Publications in Climatology, v.8, n.1 